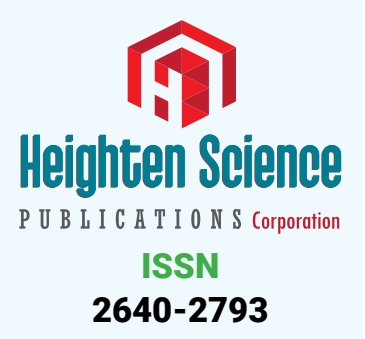

*Address for Correspondence: Mauro luisetto, Applied Pharmacologist, Independent Researcher, Italy, Tel: +393402479620; Email: maurolu65@gmail.com

Submitted: 10 April 2018

Approved: 24 April 2018

Published: 25 April 2018

Copyright: @ 2018 luisetto $\mathrm{M}$, et al. This is an open access article distributed under the Creative Commons Attribution License, which permits unrestricted use, distribution, and reproduction in any medium, provided the original work is properly cited

Keywords: BPH; Medicinal chemistry; Clinical pharmacy; Drug design; Drug delivery

Check for updates
Research Article

\section{The Immunitary role in chronic prostatitis and growth factors as promoter of BPH}

Mauro luisetto ${ }^{1 *}$, Behzad Nili-Ahmadabadi², Ghulam Rasool

Mashori ${ }^{3}$, Ram Kumar Sahư ${ }^{4}$, Farhan Ahmad Khan ${ }^{5}$, Cabianca luca $^{6}$ and Heba Nasser ${ }^{7}$

${ }^{1}$ Applied Pharmacologist, Independent Researcher, Italy

${ }^{2}$ Pharm D/PhD innovative Pharmaceutical Product Development Specialist, USA

${ }^{3}$ Professor \& Director, Department of Medical \& Health Sciences for Woman, Peoples University

of Medical and Health Sciences for Women, Pakistan

${ }^{4}$ Assitant Professor Columbia Institute of Pharmacy, Tekari, Raipur (CG)-493111, India

${ }^{5}$ Professor \& Head, Department of Pharmacology, AIMSRC, Udaipur, Rajasthan, India

${ }^{6}$ Biomedical Laboratory, Turin, Italy

${ }^{7}$ Pharm D/PhD in Microbiology \& Immunology, Faculty Member at Heliopolis University, Italy

\section{Abstract}

In the actual medical therapy of BPH, we can see: antibiotics, alpha blockers, 5-ARI, fitotherapeutics/natural products (Serenoa repens) with different which display clinical activities and other molecules such as FANS (local or systemic dosage forms) cortisones and others. Relationship between immune systems and chronic prostatitis are strictly involved in BPH progression. A vicious cycle that involve chronic flogosis, tissue remodeling, grow factors, inhibition of apoptosis, and other phenomena. Observing BPH pathogenesis under an immunologic point of view make possible to search new pharmacological strategies, to improve actual therapy.

The aim of this work is to observe some relevant literature in our opinion related the management of BHP and its progression under a pharmaceutical and immunological point of view. A deep knowledge in the pharmaceutical properties of some molecules (antimicrobials, antiphlogosis agents, Anti-androgenic agents, alpha blockers, 5-ARI and other treatments, techniques, interventions or instruments) can help the physicians to pick the right choice.

\section{Introduction}

BPH Benign prostate hyperplasia is currently consider a common progressive enlargement of the prostate gland caused by a benign overgrowth of chiefly glandular tissue that occurs especially in some men over 50 years old and that tends to obstruct urination by constricting the urethra with heavy symptomatology and related consequences in quality of life.

The current medical therapy (based on 5-ARI, alfa blokers, antimicrobials, fitotherapics, anti flogosys Antiedemigen and other) can and must be improved to delay or avoid surgery. In order to introduce new pharmacological strategy is crucial to observe the immune implications in BPH. Reports in biomedical litrerature indicate that in BPH progression some of the following factors are heavily involved:

1. Bacterial/ viral prosthetic infections

2. Chronic phlogosis 
3. Hormonal factors

4. Autoimmune reactions,

5. Urinary reflux

6. Diet

7. Seating bathroom habits/behavior, sedentariety

8. Pelvic congestion

9. Proctities

10. Prostate ducts obstruction

\section{Microcalcification}

All these events contribute to an increased production of phlogosis mediators, growth factors (stromal and epithelial) in a vicious cycle and as a result, we would have an increase and complication of the pathogenic conditions. The presence of immune system in prostate prostate gland is very well alive and manifest itself in different forms, from infiltration of lymphocytes $\mathrm{T}$ and macrophages caused by phlogosis to cytokines that cause hypertrophy. These reactions along with cellular damage produce ROS reactive oxygen species, increase in production of growth factors (VGEF, IL 8 FGF 7, TGFB, FGF 2 and others), inhibition of apoptosis and tissue remodelling with hyperproduction of extracellular matrix and stromal parts.

Other factor that can be responsible in the relapse in chronic prostatitis in BPH are microcalcifications and obstruction of the ducts. But it is not all over, there are still other conditions that can be involved, such as metabolic syndrome, diabetes and the related biochemical and hormonal factors that can modify methabolisms that can worsen the pathogenesis. Many other factors still play in. Example are factors, such as the command-and-control of the nervous systems over the bladder, uretral restrictions and other Urological disease can cause important complications. Also the creation of a biofilm over the prostate capsule makes the penetration of several antimicrobial agents look like resistant which reduces their prostatic influx. Be patient it's not over yet, we can still mention complications caused by such as IPB, urinary reflux (chemical cystitis and prostatitis), prostate cancer and other factors. But those are not the only factors, there are also often Intestinal functional abnormalities and pathologies, anorectal, sexually transmitted infectious comma as well as complications due to bacterical sanctuaries and intraprostatic calcifications.

We have seen that a bacterial infection can be primary or secondary (bacteria that causes infections or superinfectious in a tissue affected by phlogosis or to other conditions), for example in hypertonic pelvic sphincter muscle. (Bacteria can come across also lymphatic way (from rectum), by emetic way, rising uretral infectious). Adrenergic ipertonus of prostate capsule contribute to BPH physiopathology. Involved often Intestinal functional abnormalities and pathologies like ano-rectal disease, sexual transmission infectious and also due to the bacterial prostate sanctuaries properties and role played by the intraprostatic calcifications.

In The actual medical therapy of BPH we can see: antibiotics, alfa blokers, 5-ARI, and fitothearapic (Serenoa repens) with different clinic functions and activities and other molecule as fans (local or systemic), cortisons et. Other.

The aim of this work is to observe some relevant literature in our opinion related the management of BPHS and progression under a pharmaceutical- Eendocrinologicmetabolic disease and immunologic point of view. A deep knowledge in the pharmaceutical 
properties of some molecule (antimiciobials, antiflogosis Antiedemigen, alfa blochers, 5- ARI and other intruments) can help the physicians in the right choice. The same pharmaceutical industries can introduce in therapy new drugs improving molecular formula or delivery systems to achieve improvement in kinetics and dynamics. Also little improvement in pharmaceutical or pharmacological molecular properties can produce relevant clinical effect.

\section{Material and Methods}

Using a review method and observing some literature in biomedical database can be produced a new approach in pharmacological strategies in some relevant urologic disease or to prevent it. Observing some works related to pathology like BPH we to try find NEW strategies that could be translated in order to achieve better clinical results adding new drug delivery system or other improvement in molecular structure in order to achieve more efficacy and reduced relapses or surgical needs (treating, controlling or even curing the root causes or to manage to lessen the security or prevent some of those role playing factors).

\section{Results}

From literature we can find:

- According V. Ficarra "In this issue of BJU International, Gandaglia et al., summarize the evidence supporting the role of chronic prostatic inflammation in the pathogenesis and progression of BPH. Briefly, one or more concomitant factors (bacterial infections, viruses, sexually transmitted organisms, dietary factors, hormones, autoimmune response and urine reflux) can stimulate an inflammatory reaction in prostatic tissue characterized by infiltration of T-lymphocytes, activation and up-regulation of pro-inflammatory cytokines, increased expression of potent stromal and epithelial growth factors (e.g. fibroblast growth factor, FGF-2) and consequently abnormal proliferation of prostatic cells. Moreover, local hypoxia plays an important role stimulating reactive oxygen species (ROS) release, Continuous repair gives tissue remodeling. Neo-vascularization processes and the production of other additional growth factors (vascular endothelial growth factor, interleukin 8, FGF-7, TGF- b and FGF-2). Interestingly, this mechanism is self-perpetuating, creating a local vicious cycle. Available clinical data seems to emphasise the prevalence of chronic prostatic inflammation in BPH. Indeed, a sub-analysis of the REDUCE (Reduction by DUtasteride of prostate Cancer Events) trial shows that in patients with $\mathrm{BPH}$ a chronic prostatic inflammation can be detected in $77 \%$ of patients who underwent prostate biopsies. Moreover, this study also showed a statistically and clinically significant correlation between chronic prostatic inflammation and LUTS severity, especially when the storage subscale was considered. As extensively described by Gandaglia et al.,., other studies have shown a significant correlation between chronic prostatic inflammation and prostate volume and an increased risk of acute urinary retention. Obviously, chronic prostatic inflammation can be histologically detected only in patients who undergo prostate biopsies for suspicion of prostate cancer. However, most patients with LUTS/BPH do not undergo a prostate biopsy. For this last category, the use of specific biomarkers correlated with chronic prostatic inflammation has been proposed as a potential alternative. Although interleukin 8 seems to be the most reliable and predictive surrogate marker to identify patients with chronic prostatic inflammation, its use is not yet popular, it is expansive and probably requires further clinical evaluation before introduction into daily clinical practice. In this context, the detection of prostatic calcifications can represent a simple ultrasound sign to suspect the presence of chronic prostatic inflammation. In patients aged $>50$ years, prostatic calcifications represent an 
age-related alteration of the prostatic fluid. Prostatic calcifications can produce an obstruction of the intraprostatic ducts stimulating an inflammatory response in prostatic tissue characterised by lymphocyte infiltration, cytokine activation and ROS release. This results in damage of epithelial and stromal prostatic cells and a subsequent process of wound healing consisting of stromal proliferation and excessive extracellular matrix production; summarises these mechanisms following prostatic duct obstruction.

I think that in patients with prostatic calcifications and severe LUTS (with predominant storage symptoms) the presence of chronic prostatic inflammation should be strongly considered. Patients with high-grade prostatic inflammation seem to have a worse response to traditional medical therapy for LUTS/BPH (a -adrenergic blockers and 5 a -reductase inhibitors) compared with patients without or with lowgrade prostatic inflammation [1]. Indeed, neither a-adrenergic blockers nor 5. A -reductase inhibitors have an anti-inflammatory effect. Therefore, drugs commonly used for the treatment of non-neurogenic LUTS cannot influence the described chronic inflammatory status. An anti-inflammatory effect on human prostate has been ascribed to the hexanic lipidosterolic extract of Serenoa repens. Specifically, in 2003 Vela Navarrete et al.,. [2] reported a significant reduction of interleukin 1 and TNFa levels 3 months after Serenoa repens treatment compared with the placebo arm. Recently, Latil et al. [3], showed that Serenoa repensinhibits the expression of two" [4].

- Hoon Choi et al., writed that "Dutasteride showed greater efficacy in reduction of TPV and PSA and in symptomatic improvementby IPSS score than finasteride [5]." See the different chemistry formula and related pharmacolgocial pfrofile of action (reversible or irreversible activity in 5-HT INIBITION) but also related to other factors as tissue penetration. Related chemical groups ad lipofilic balances.

- Luisetto et al., "It is clearly known that the prostatic gland is frequently involved in Different pathologies in adults and elderly patients. Benignant or malignant: anatomic of functionally disease. Frequently other condition as bladder dysfunctions can be associated or added to this pathologies or causated by it. Some of these pathologies give low level in patient quality life and reducing in life expectance (malignant). Malignant pathologies start as local disease but can diffused as metastatic interesting other apparatus of the patient (patient frequently show resistance to first line therapy in example hormonal blocks, or different chemiotherapic). But In all of this pathology we can see a not complete resolution with current medical therapy in all patients and this can be related with the difficulties of some drugs to penetrate adequately in the tissue. In example we can see that I many benignant cases as IPERTROPHIA also fitotherapic produces or dietetic integrator are frequently added by the specialist to the classic drugs politherapy. This shows that the classic drug therapy can be improved. In example Relapses in bacterial chronic prostatitis are commonly in great number of patient even under the best pharmacological therapies available today. It means that something goes wrong in this kind of therapy [6].

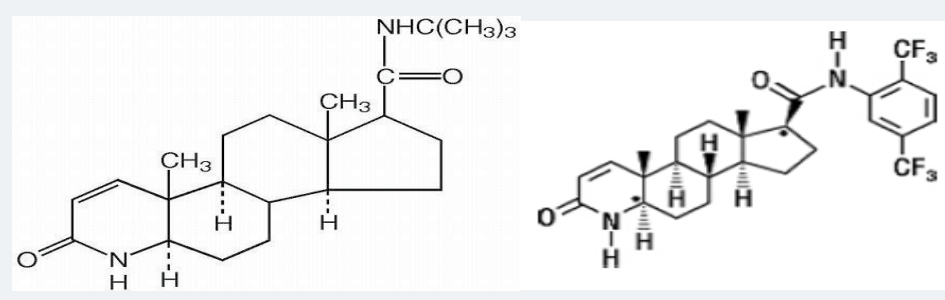

(Remember that flogosis can results in partial inefficacy of 5-AR1) FINASTERIDE DUTASTERIDE. 
- "According Biomedical Literature luisetto et al., "Responsible of relapses of chronic prostatitic and related relapses can be: biofilm, reduced urinary prostatic fluss in example by IPB, urinary reflux (chemical cystitis and prostatitis), prostatic cancer and other factors.

a. Biofilm is responsible about difficulties in diagnostic methods and in therapy.

b. Bacterial prostatitis is associated in frequent E. Coli infections, enterococcus faecalis but other germs

c. Can be involved (example clamidia, mycoplasma).

d. Involved often Intestinal functional abnormalities and pathologies, ano-rectal, sexual transmission infectious and also due to bacterial sanctuaries and intraprostatic calcifications.

e. We have see that a bacterial disease can be primitive or secondary (bacteria cause of infection or sovra infectious in a flogosis tissue or to other conditions) in example ipertonus in pelvic sfinteric muscle.

f. We have see the that infectious can be across different process: Trought ematogenic, lymphatic, contiguity, local pathology, sexual way, trans uretral UTI, GI pathologies, proctities, neoplasia, prostatic reflux, rectal bacteria linfatic diffusion and other.

g. Medical examination and Ecografy make possible to evaluate local situation in order to exclude other pathologies.

h. Ascessual evolution, micro litiasis and other tissue abnormalities.

i. Acute disease can develop in chronic evolution, with ascessual possibilities (needed surgical drainage) Only the Antimicrobial therapy efficacy only in little prostatic ascessual Cavity. Prostatic Ascess Contribute to Obstruction [4].

Vela Navarrete $\mathrm{R}$ et al., write that "The role of infiltrating cells (I.C.), commonly observed in the adenoma interstitial tissue, is unknown. We tested the hypothesis that I.C. are related with BPH progression by: phenotypically characterizing these cells; quantifying the expression of lymphokines and growth factors; investigating the response to Permixon (P) in a clinical study. Permixon is a lipido sterolic extract of Serenoa repens possessing pharmacological activities and widely used in the treatment of men with BPH.

\section{Histological}

A difference was observed in the number of lymphocytes B between $C(91.4+/-44.1)$ and $P$ treated $(58.2+/-53.7)$ groups $(\mathrm{p}=0.097)$. BIOLOGICAL MARKERS: TNFalpha and IL-1beta were dramatically lower in the Permixon treated group. Other parameters did not show significant changes. CLINICAL: IPSS in the Permixon treated group was significantly reduced $(\mathrm{p}<0.006)$ from $20.0+5.9$ to $14.9+3.8$ after three months of treatment.

The BPH inflammatory hypothesis was tested in humans. Our pilot study shows a significant reduction of some inflammatory parameters in prostatic tissues of patients treated with Permixon. These biological findings justify a pharmacological effect of this drug on the inflammatory status of the adenoma. A correlation with clinical improvement was observed [5].

Latil A et al., "What's known on the subject? And what does the study add? Pervasive inflammatory infiltrates, mainly composed of chronically activated $\mathrm{T}$ cells and monocytes/macrophages, have been observed in benign prostatic hyperplasia 
(BPH). Permixon ${ }^{\circledR}$, a hexanic lipidosterolic extract of Serenoa repens (hexanic LSESr) used to treat urinary dysfunction in BPH patients, has anti-inflammatory activities. This paper provides new insights into the anti-inflammatory properties of Permixon ${ }^{\circledR}$. We report that hexanic LSESr inhibits early steps of leukocyte infiltration in vitro by downregulating MCP-1/CCL2 and VCAM-1 expression.

To investigate the mechanisms by which hexanic lipidosterolic extract of Serenoa repens (hexanic LSESr) may prevent leukocyte infiltration in benign prostatic hyperplasia by studying its impact on monocyte chemoattractant protein 1/ chemokine (C-C motif) ligand 2 (MCP-1/CCL2) and vascular cell adhesion molecule 1 (VCAM-1) expression in vitro. After pretreatment with hexanic LSESr, human prostate (epithelial and myofibroblastic) cells and vascular endothelial cells were stimulated with proinflammatory cytokines. MCP-1/CCL2 and VCAM-1 mRNA expression was quantified by real-time PCR. ELISA kits were used to determine MCP-1/CCL2 levels in culture supernatants and VCAM-1 expression in living cells.

Hexanic LSESr reduced MCP-1/CCL2 mRNA levels in both epithelial (BPH-1) and myofibroblastic (WPMY-1) prostate cell lines. Hexanic LSESr downregulated MCP1/ CCL2 secretion by WPMY-1 cells in a concentration-dependent manner, more efficiently than Serenoa repens extracts obtained by supercritical carbon dioxide extraction. Hexanic LSESr inhibited tumour-necrosis-factor- $\alpha$-induced MCP-1/CCL2 secretion by the human vascular endothelial cell line EAhy.926, as well as surface VCAM-1 protein expression, in a concentration-dependent manner. Hexanic LSESr impedes key steps of monocyte and $\mathrm{T}$ cell attraction and adherence by inhibiting MCP-1/CCL2 and VCAM1 expression by human prostate and vascular cells in an inflammatory environment. These findings provide new insights into the anti-inflammatory effects of the hexanic lipidosterolic extract of Serenoa repens, Permixon ${ }^{\circledR}$, in benign prostatic hyperplasia [6].

- Skeldon SC et al., "We investigated the risk of cardiovascular events among patients receiving dutasteride relative to finasteride. We performed a population based cohort study of Ontario men 66 years old or older who commenced treatment with dutasteride or finasteride between October 1, 2005 and March 31, 2015. For each individual treated with dutasteride, we identified 1 treated with finasteride, matching on a propensity score and calendar quarter of treatment initiation to account for temporal changes in prescribing. The primary outcome was hospitalization for heart failure. Secondary analyses were done to examine acute myocardial infarction and stroke. Cox proportional hazards regression was used to adjust for differences between groups.

We studied 36,311 men who commenced dutasteride and 36,311 treated with finasteride. In the primary analysis, we found no difference in the risk of heart failure among patients receiving dutasteride relative to those receiving finasteride (adjusted HR 0.98, 95\% CI 0.88-1.08). Similarly, we found no difference in the risk of acute myocardial infarction (HR $0.94,95 \%$ CI $0.82-1.08$ ) or stroke (HR 1.03, 95\% CI $0.88-1.20$ ). In this population based cohort study of more than 72,000 older men, dutasteride was not associated with an increased risk of cardiovascular events relative to finasteride [7].

- According Loke YK et al., "A recently published large, long-term randomized controlled trial (RCT) brought into question the safety of dutasteride after a significantly increased risk of 'cardiac failure' was noted in the dutasteride arm of the trial compared with placebo. Our objective was to perform a meta-analysis to assess the risk of cardiovascular adverse events with the use of dutasteride for the prevention or treatment of prostatic disease. We searched MEDLINE and EMBASE, unpublished articles identified through FDA/EMEA websites, study registers of pharmaceutical companies and reference lists of articles. Parallelgroup, randomized controlled trials where men received dutasteride for the 
prevention of prostate cancer or treatment of prostatic hyperplasia against any comparator intervention were included. Heart failure was the primary outcome of interest but we also looked at myocardial infarction and stroke. Fixed-effect meta-analysis of pooled relative risk (RR) ratios of adverse effect outcomes was conducted.

In all, 12 RCTs were included in the meta-analysis after detailed screening of 564 citations. The total number of participants was 18,802, and study duration ranged from 6 to 208 weeks. Only two trials provided details on adequate allocation concealment, whereas all the trials stated they were double blind in nature. Dutasteride was not associated with a statistically significant increased risk of heart failure (RR $1 \cdot 05 ; 95 \%$ confidence interval [CI], 0.71-1.57, I $(2)=20 \%$ ), myocardial infarction (RR 1.00; 95\% CI $0 \cdot 77-1 \cdot 30, \mathrm{I}(2)=0 \%)$ and stroke $(\mathrm{RR}, 1 \cdot 20 ; 95 \% \mathrm{CI} 0 \cdot 88-1 \cdot 64, \mathrm{I}(2)=0 \%)$ as compared to controls. We did not find consistent evidence of a significant association between dutasteride therapy and the risk of cardiovascular adverse events [8].

- I. R. Ravish et al., "Dutasteride significantly improved Qmax, reduced IPSS score, and improved Quality of Life as compared to Finasteride at the end of the 12-week period. Dutasteride with its inhibitory effects on type 1 and 25 alfa reductase, produces significantly better results than Finasterid [9].

- P.TOREN Et al., "On multivariable logistic regression analysis, dutasteride was the only factor that significantly reduced clinical progression of benign prostatic hyperplasia, with an odds ratio of 0.47 (95\% confidence interval 0.37 to 0.59 , $\mathrm{P}<0.001)$ " [10].

- Liptay S1 et al., writed that "1. Chronic inflammatory diseases have been shown to be associated with NF-kappaB activation and impaired apoptosis of immune cells. The aim of the present study was to investigate if sulfasalazine and its colonic metabolites 5-aminosalicylic acid (5ASA) and sulfapyridine affect NFkappaB/Rel activation and viability of T-lymphocytes. 2. Sulfasalazine inhibits NF-kappaB/Rel activation in the murine T-lymphocyte cell line RBL5 using electrophoretic mobility shift assays. In transfection assays sulfasalazine treatment for $4 \mathrm{~h}$ inhibits kappaB-dependent transcription with an IC50 value of approximately $0.625 \mathrm{mM}$. 3. Higher doses or prolonged treatment result in cell death of T-lymphocytes in a dose- and time-dependent manner. Cell death is caused by apoptosis as judged by DNA fragmentation, annexin V and Apo 2.7 staining. Induction of apoptosis is a fast event with $50 \%$ apoptotic cells after a $4 \mathrm{~h}$ incubation with $2.5 \mathrm{mM}$ sulfasalazine. The ED50 value for apoptosis induction after $24 \mathrm{~h}$ treatment was approximately $0.625 \mathrm{mM}$. 4. In contrast, 5ASA and sulfapyridine neither inhibit NF-kappaB/Rel activation nor induce apoptosis in T-lymphocytes at doses up to $5.0 \mathrm{mM}$. 5. These results demonstrate that sulfasalazine, but not 5ASA or sulfapyridine, strongly inhibits NF-kappaB activation and potently induces apoptosis in T-lymphocytes. Inhibition of NFkappaB/Rel activation and subsequent clearance of activated T-lymphocytes by apoptosis might thus explain the beneficial effects of sulfasalazine in the treatment of chronic inflammatory disorders [11].

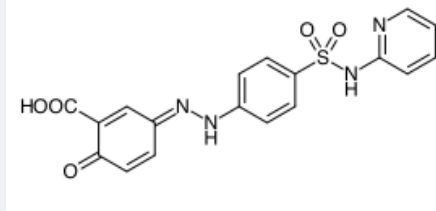

Sulfasalazin
Dickdarm-

bakterien

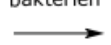

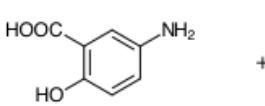

Mesalazin

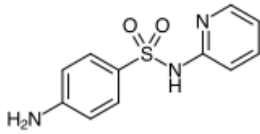

Sulfapyridin

Mesalazin (inibit nf-kb - induce apoptosis in linph. T) 
See also in example: United States US 20040198826A1 (12) Patent Application Publication (10) Pub. No.: US 2004/0198826A1 Baiker et al. (43), Pub. Date: Oct. 7, 2004 PHARMACEUTICAL COMBINATION FOR TREATING BENIGN PROSTATIC HYPERPLASA OR FOR TREATING ABACTERIAL PROSTATITIS: "0018 Within the meaning of the present invention, preferred non-Steroidal inflammatory drugs are the cyclo-oxy. US 2004/0198826 A1genase (COX) inhibitors, such as the non-selective COX inhibitors acetylsalicyclic acid, mesalazin, ibuprofen, naproxen, flurbiprofen, fenoprofen, fenbufen, ketoprofen \& other.

Perletti G et al., "Chronic bacterial prostatitis (CBP) is a persistent infection of the prostate characterized by poor quality of life mainly due to frequent relapse episodes caused by incomplete eradication of causative pathogens. Aggressive antibacterial therapy is required to attenuate the severe symptoms of CBP and to achieve a permanent cure. Although fluoroquinolones are currently recommended as first-choice agents, macrolide antibiotics are emerging as a noteworthy option for the treatment of CBP. Macrolide antibiotics are characterized by an impressive array of distinct pharmacokinetic (PK) and pharmacodynamic (PD) properties. These properties include high intracellular accumulation in phagocytes and at sites of infection, including the prostate; broad antibiotic but also biofilm-inhibiting properties; immunomodulating and inflammation-resolving activities. These features offer particular advantages for the treatment of chronic infections of the prostate gland, which are not easily amenable to drug therapy. Macrolides may be exploited to counteract the unsatisfactory rates of clinical symptom improvement and pathogen eradication. The results of a number of clinical trials support this proposal [12].

Ikeuchi $\mathrm{T}$ et al., "We analyzed the incidence of anal disease in patients with nonbacterial prostatitis (NBP) or with prostatitis-like syndrome (PLS), and evaluated the clinical efficacy. The complicated rate of anal disease in these patients was $29.7 \%$ (31.8\% for NBP and $28.1 \%$ for PLS), and the overall incidence of active anal disease was $15.4 \%$ (16.2\% for NBP and $14.8 \%$ for PLS), it yielded a significantly higher complicated rate than other urological disease ( $p$ less than 0.01 ). The most common type of anal disease was hemorrhoids, especially piles. The clinical cure rate for anal disease in NBP patients was $71.4 \%$, and in PLS patients was 58.2\%. The high incidence of hemorrhoids (especially piles) was in these patients by clinico-statistical observation suggests that the development of anal disease may be etiologically correlated with NBP and PLS. Furthermore, we noted that Kampo treatment (Keisibukuryogan) was useful in the treatment of prostatitis complicated by anal disease, especially when combined with anti-hemorrhoidal suppositories against active anal disease in PLS patients ( $p$ less than 0.05) [13].

And according Takechi $\mathrm{S}$ et al., "The pathogenesis of nonbacterial prostatitis (NBP) is not understood mainly due to the lack of appropriate experimental models. We developed a new experimental model of NBP by inducing a partial obstruction of the urethra (PUO) in the rat. Male Wistar rats aged 12 weeks were used. PUO was produced by a nylon ligature on the urethra over a rubber tube. The tube was slipped out after the ligature had been tied. Two rats were examined histologically $6 \mathrm{~h}, 1$ day, 3 days and 7 days after PUO. In another group, two rats were killed at 1, 3 and 7 days after the release of the PUO that had been left in place for 3 days. On day 3 , another eight rats with PUO and eight control rats had $2 \mathrm{ml}$ of urine in the bladder replaced by the same volume of lucifer yellow (LY; $10 \mathrm{microg} / \mathrm{ml}$, MW 500), microperoxidase (MP; $20 \mathrm{microg} / \mathrm{ml}$, MW 1900), horseradish peroxidase (HRP; $10 \mathrm{microg} / \mathrm{ml}$, MW 40 000), or saline as control, respectively. Lymphocytic infiltration and interstitial edema were noted in the prostate following PUO, being most prominent on day 3. After the release of the PUO, these inflammatory changes gradually disappeared. Only LY was noted within the prostatic stroma of the rats $2 \mathrm{~h}$ after bladder instillation. Intraprostatic urinary reflux may be an etiologic factor in NBP. The present study showed that lower 
urinary tract obstruction caused NBP in the rat. Penetration of prostatic tissue by lowmolecular-weight substances in the urine may trigger NBP [14].

\section{Discussion}

In BHP we can observe a great damage to the patient quality of life and cost for public institutions and insurance. We must consider that BHP disease under an infective, endocrine and immune phenomena deeply interconnected. Non pharmacological strategies can be Kegel exercise to reduce pelvic ipertonus, lifestyle, dietary, physical activity and other factor can reduce relapsis in chronic and acute prostatitis related in $\mathrm{BPH}$, reduction of pelvic congestion. No sedentariety, no stinging species, no etilic alcohol drinks, no cured meats et other. The Intestinal flora situation is relevant to prevent exchange of bacteria form rectum, proctological disease, hemorrhoids.

In relapses of BHP as chronic or acute prostatitis factors like different kind of bacteria population in the infectious and related activity profile of antimicrobial used ( gram+, gram -, mycoplasma and calmidya, herpes virus), profile of resistance, biofilm presence, tissue iperplasia, chronic flogosis, autoimmunity, kinetics of tissue penetration etc. must be high considered to achieve the best results. Also the receptor local situation in bladder and trigon (adrenergic, cholinergic) are relevant factors useful in therapy strategy to improve urinary flow (as wee as anatomic obstruction of prostate uretra) and other.

Under the light o reported literature Prostate gland must be considered an immunology's subject in some disease at BHP and chronic prostatitis and this aspect must not to be overlooked especially in therapy. In resistances profile other antimicrobials as, ampicillin, carbenicillin, doxicillin, gentamicin, imipenem, piperacillina tazob, Fosfomicin parenteral and other. In this paper we have see only some molecule currently in use to show the relationship between dynamics, kinetics, drug delivery strategy and medicinal chemistry properties. Prostate gland is considered a pharmacological sanctuaries and for this reason using the best strategies it can result in more \% of global clinical efficacy. Rigth spectrum anti-microbial covering, right time of the cure, the right antiflogosis and anti edemigen therapy associated to 5- ARI to induce apoptosis and alfa blockers can be the golden endpoint to prevent the progression of BHP. Also a good blood glucose control prevent recurrent urinary infectious (glucose is normally used by microbs growth) and uric acid blood monitoring and control and therapy can be useful to reduce global stinging Situation (crystals).

Observing some of these works related to some pathological conditions like BPHP in attempt to find NEW treatment strategies that could be translated in new efficient treatments in order to achieve much better enhanced clinical results, such as: New therapeutical combinatorial cocktail regimen.

Drug delivery systems, achieving more efficacy and reduced both side effects and relapses or surgical needs:

1. innovative locoregional or targeted drug delivery systems with higher prostatic<smiles>O=C(O)c1cn(C2CC2)c2cc(N3CCNCC3)c(F)cc2c1=O</smiles>

Examples of some antimicrobials used (only few examples). 

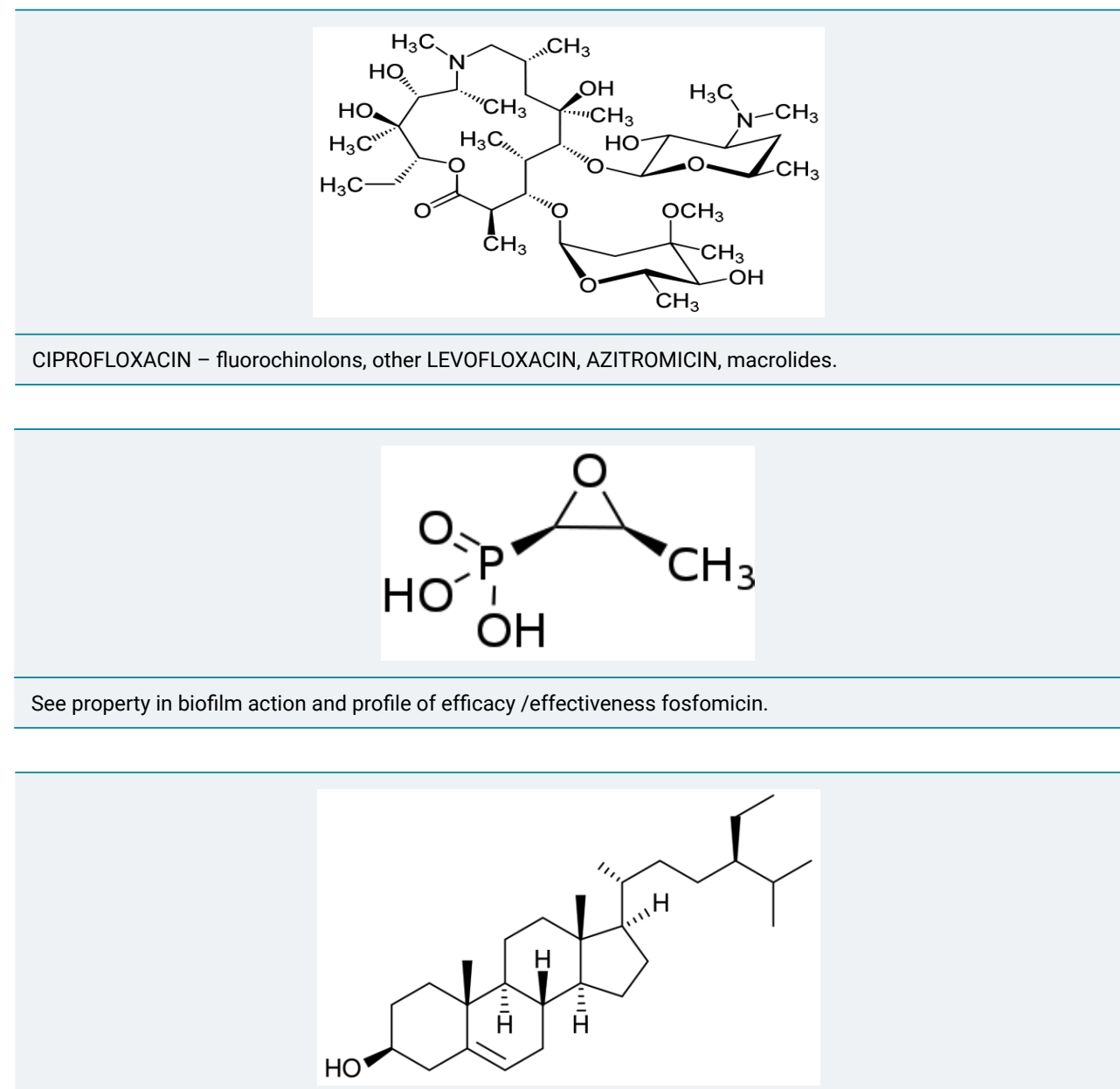

SERENOA REPENS alcaloids Beta fitosterols.

1a. intra- or extra-capillary

2a. or even intratissue drug

2. or combination of new drug regimen levels with not only

2a. more localized but even

2b. more extended drug levels present for longer periods of time in the target tissues

3. drug delivery systems can be also used for

3a. more than one drug or

3b. the use of more than one which each of them releases a specific drug, which yields a combinatorial treatment in the same dosage form the Canby

3b1. colloidal micro- or nanosystems, alone

3b2. or the combination of colloidal systems with polymeric implant systems, using minimally invasive methods. Obviously once we have a both localized and combinatorial treatment, we would need less drug levels, because we can both bypass all the barriers, by modifying biopharmaceutical as well as pharmacokinetic conditions on removing many barriers, establishing more stable levels which by consequence reduces the risk of relapse and at the same time we could much more efficiently manage the pathological conditions of the very complicated multifactorial disease in much more efficient way, and obviously pharmacokinetics means a lot when it comes 
to for instance the penetration of antibacterial antiviral drugs or bypassing the biofilm capsule or when we have already less inflammation, obviously we would have less hypertrophy, and consequently and obviously we will logically need less dosage and drug levels in each case.

4. Moreover once the drug is released locoregionally, more safe type of molecules for example

4a. antibacterial

4b. antiviral or

4c. even anti inflammatory responses can be used or even improvements in by manipulating or modifying the molecular structure in order to render the molecule more potent do an increased hydrophobicity or even specific receptor affinity while avoiding less toxic structures such as the presence of extremely molecular moieties with in some cases very strong electrophilic nature and a tendency to create covalent bonds with DNA [15], achieving much less toxic and more potent drugs which is translated in more efficacy and reduced both side effects and relapses or the need surgical interventions.

\section{Conclusion}

From literature we can see some relevant implication in pharmacological therapy related to medicinal chemistry, pharmacological or delivery system properties of some molecule currently in use. In medical approach the right profile of action (dynamics, antibacterial spectrum, dosage, duration of therapy, pharmacokinetics, tissue penetration and other characteristics) are to take in great consideration in this kind of situation. Even little modify in kinetics, dynamics or delivery system and sequential approach (for antimicrobials) can produce improve in clinical outcomes in BPH medical therapy and control. Drugs strategies that must consider antimicrobial power, antimicrobial concentration, alfa bloccants effect, 5 HT inhibitions, anti flogosis but also new drug delivery systems. Andi edemigen, antioxidant et other as well as preventive non pharmacological strategies like right water intake, physical activity, no traumatic sports, not sexual abuse but regular (spermatozoa degradation products), and other From literature we have see the preminent role played in BPH by chronic flogosis,as well as hormonal umbalancec that produce tissue remodeling, preproduction of growth factors and reduced apoptosis.

\section{All factors involved in BHP progression}

So a deep knowledge in medicinal chemistry of molecule currently used make possible a more rational therapy of chronic prostatitis as well as BHP as well as to introduce by pharmaceutical industries improved or new molecule with a better profile of action. What is relevant is that all chronic prostatitis must be treated in the right way since first time to prevent chronic progression and the vicious circle. Can we think new delivery system to achieve better improvement in local tissue penetration of Therapeutics principles?

We know that it clear that the drugs vehicle is important as the same drugs and that modifying the molecule we can improve the activity in relevant way also due by different tissue diffusion (In example see the chinolons and fluoro chinolons different pattern of tissue penetration) and in duration of action time

As request to prevent relapses in acute and chronic prostatitis.

Lipophilic hydrophilic balances, acid-bases properties, molecular weight and other pharmaceutical characteristics great influence the pk. Kinetics as VD or T1/2 but also the profile of linked chemical groups can give new delivery of classic drugs (Other can 
be acid or basic molecular properties or velocity in metabolism). We know that for example in the same drug classes in examples chinolons little chemistry modify can results in improvement of efficacy so we can think that little chemical modify o new delivery system can produce better clinical results by a better pharmacokinetics in this sanctuaries.

We can consider innovative drug delivery systems to improve clinical efficacy reducing global toxicity. Novel loco-regional drug delivery systems or more targeted to prostatic tissue, more time extended drug. Presence in prostatic tissue, using of innovative technologies as colloidal micro- Nano systems and other, better biofilm discgregation properties, modifying lipofilic-idrofilic molecular balances, acid bases properties of molecule, add of more useful chemical groups to classic formula to improve kinetics and prostatic intake, implants, and other medicinal chemistry new technologies that can add advantages vs the actual therapy models.

All this strategies must be added to adeguate flogosys control and towards preventing linphocite $\mathrm{T}$ and macrophages prostate infiltration and an efficacy grow factors control.

\section{Clarifications}

This paper was not written for any diagnostic or therapeutic intent, only to produce new research hypotesys.

\section{References}

1. Luisetto M, Nili-Ahmadabad B, Mashori GR. Relapses and Recurrent Chronic Bacteria Prostatitis Biofilm Related, A Case Report. J Pharmacology Clin Res. 2017; 4. Ref.: https://goo.gl/zJ68rs

2. Vela NR, Garcia CJV, Barat A, Manzarbeitia F, Lopez FA. BPH and inflammation: pharmacological effects of Permixon on histological and molecular inflammatory markers. Results of a double blind pilot clinical assay. Eur Urol. 2003; 44: 549-555. Ref.: https://goo.gl/r9zpij

3. Latil A, Libon C, Templier M, Junquero D, Lantoine-Adam F, et al... Hexanic lipidosterolic extract of Serenoa repens inhibits the expression of two key inflammatory mediators, MCP-1/CCL2 and VCAM1, in vitro. BJU Int. 2012; 110: 301-307. Ref.: https://goo.gl/1xXvHX

4. Ficarra $\mathrm{V}$. Chronic prostatic inflammation a new target in the medical therapy of lower urinary tract symptoms (LUTS) due to benign prostate hyperplasia (BPH)?. BJU Int. 2013; 112: 421-422. Ref.: https://goo.gl/1FRfQC

5. Choi H, Chang YS, Park BH, Ko DH, Kim JB, et al.,. Comparison of Clinical Efficacy of Finasteride and Dutasteride as 5-alpha Reductase Inhibitor. Korean J Androl. 2012; 30: 45-51. Ref.: https://goo.gl/zy8Uq5

6. Luisetto M, Nili-Ahmadabadi B. Chronic Prostatitis: The Clinical Pharmacist Role and New Delivery Systems. J Bioanalysis Biomedicine. 2017; 09. Ref.: https://goo.gl/fuqawC

7. Skeldon SC, Macdonald EM, Law MR, Huang A, Paterson JM, et al.,. The Cardiovascular Safety of Dutasteride. J Urol. 2017; 197: 1309-1314. Ref.: https://goo.gl/S6rxki

8. Loke YK, Ho R, Smith M, Wong O, Sandhu M, et al.,. Systematic review evaluating cardiovascular events of the 5-alpha reductase inhibitor - Dutasteride. J Clin Pharm Ther. 2013; 38: 405-415. Ref.: https://goo.gl/kkNHR4

9. Ravish IR, Nerli RB, Amarkhed SS. Finasteride to Evaluate the Efficacy of Dutasteride in the Management of Patients with Lower Urinary Tract Symptoms and Enlarged Prostate, Arch Androl. 2007; 53: 17-20. Ref.: https://goo.gl/8VQz3b

10. Toren P, Margel D, Kulkarni G, Finelli A, Zlotta A, et al.,. Effect of dutasteride on clinical progression of benign prostatic hyperplasia in asymptomatic men with enlarged prostate: a post hoc analysis of the REDUCE study. BMJ. 2013; 15: 345. Ref.: https://goo.gl/xdXttB

11. Liptay S, Bachem M, Häcker G, Adler G, Debatin KM, et al.,. Inhibition of nuclear factor kappa B and induction of apoptosis in T-lymphocytes by sulfasalazine. Br J Pharmacol. 1999; 128: 1361-1369. Ref.: https://goo.gl/3xXVxa

12. Perletti G, Skerk V, Magri V, Markotic A, Mazzoli S, et al.,. Macrolides for the treatment of chronic bacterial prostatitis: an effective application of their unique pharmacokinetic and pharmacodynamic profile (Review). Mol Med Rep. 2011; 4: 1035-1044. Ref.: https://goo.gl/kXsXon 
13. Ikeuchi T, Ueno M, Yogi S, Hasegawa K, Sasaki H, et al.,. Clinical studies on chronic prostatitis and prostatitis-like syndrome. (5) Evaluation of prostatitis complicated by anal disease. Hinyokika Kiyo. 1991; 37: 1677-1682. Ref.: https://goo.gl/VwWCJe

14. Takechi S, Yokoyama M, Tanji N, Nishio S, Araki N. Nonbacterial prostatitis caused by partial urethral obstruction in the rat. Urol Res. 1999; 27: 346-350. Ref.: https://goo.gl/ZT6whz

15. Wilhelm EA1, Jesse CR, Nogueira CW, Savegnago L. Introduction of trifluoromethyl group into diphenyl diselenide molecule alters its toxicity and protective effect against damage induced by 2-nitropropane in rats. Exp Toxicol Pathol. 2009; 61: 197-203. Ref.: https://goo.gl/VUjWir 